\title{
Maintenance-Oriented Design in Architecture. A Decision Support System for the Evaluation of Maintenance Scenarios Through Bayesian Networks Use. A Case Study: the Headquarters of ING Groupe in Amsterdam
}

\author{
Michele Di Sivo ${ }^{1}$, Daniela Ladiana ${ }^{2}$, Federico Novi ${ }^{2}$ and Caterina Salvatori ${ }^{2}$ \\ ${ }^{1}$ Dipartimento di Ingegneria dell'Energia, dei Sistemi, del Territorio e delle Costruzioni \\ dell'Università di Pisa (DESTEC), Largo Lucio Lazzarino, 2, 56122 - Pisa, Italy, \\ michele.disivo@unipi.it. \\ 2 Dipartimento di Architettura di Pescara, Università degli Studi “G. d'Annunzio" di Chieti e Pescara, \\ Viale Pindaro, 42, 65127 - Pescara, Italia \\ d.ladiana@unich.it, fn@officinadiarchitettura.net, caterinasalvatori@libero.it
}

\begin{abstract}
This study was developed at the Department of Architecture of Pescara and Department of Energy, Systems, Territory and Construction Engineering of the University of Pisa as part of an experimental thesis that led to the implementation of a Decision Support System. The objective of the work was to implement a tool capable of evaluating - in relation to the choices concerning the morphology of the building, the construction technologies, the materials and the design of the architectural elements - the levels of maintenance quality implemented in the various phases of the project, from the first phases, in which few relevant decisions are made, to the executive phase characterized by a multiplicity of choices. The aim was to construct a tool in which the reliability of the evaluations was related to the quantity and quality of the data that feeds the decision-making process, but which is also able to evaluate preliminary decisions based on the elements of choice that characterize the first phases of the project. The conceptual model has been defined through the construction and implementation of a Bayesian Network or a graphical system of probabilistic inference able to represent the set of stochastic variables and their conditional dependencies through the use of a direct acyclic graph. Through the interrogation of the network it is therefore possible to evaluate through the expression of a synthetic index, a real overall rating of the different aspects that contribute to define the maintenance quality. The use of Bayesian Networks, in the light of the analyses carried out on an experimental basis - exemplified here on the case study of ING Groupe headquarters - for the ability to control a multitude of factors linked to the durability of materials, the morphology of systems and ease of intervention, seems capable of generating useful, effective and expandable tools to support the design decision-making process.
\end{abstract}

Keywords: Design, Durability, Maintenability, SSD, Bayesian Networks.

\section{Introduction}

The architectural design of buildings is increasingly characterized by a new morphological, functional and technological complexity that requires, from coordination to control, maintenance and management of the entire life cycle, new skills to effectively implement a process in which the act of conception and the moment of construction are continuously intertwined over time (Daniotti, 2007; Latouche, 2015; Di Sivo, 1994; Ladiana, 2009).

The use of Bayesian Networks is in this experimentation proposed for the construction of 
an open model of a useful tool to manage uncertainty through the creation of conceptual maps of reality that aim to highlight the variables and links in a clear and rigorous way.

The study described in this paper has been developed at the Department of Architecture of Pescara with and the Department of Energy, Systems, Territory and Construction Engineering of the University of Pisa, starting from an experimental thesis that led to the implementation of a Decision Support System (SSD).

For the tool described, in addition to the purposes related to the operating mode and the possible implementation of databases, there is the structural purpose of being able to operate within an "open system" that can be progressively expanded, on which it would be possible, as the study and experimentation progresses, to graft new areas of evaluation, increasing its operations.

\section{Research Objectives and Methodological Approach}

The objective of the research developed was to implement a tool capable of evaluating - in relation to the choices concerning the morphology of the building, the construction technologies, the materials and the design of the architectural elements - the levels of maintenance quality implemented in the various phases of the project, from the first phases, in which few relevant decisions are made, to the executive phase characterized by a multiplicity of choices (Di Sivo, 1994).

It was intended to build a tool in which the reliability of the evaluations would be related

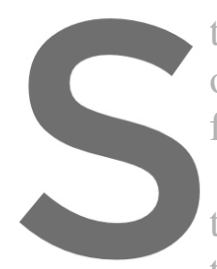

to the quantity and qua

operate also in a preliminary

first phases of the project.

The fundamental ai

to the logic of design

the scientific culture (
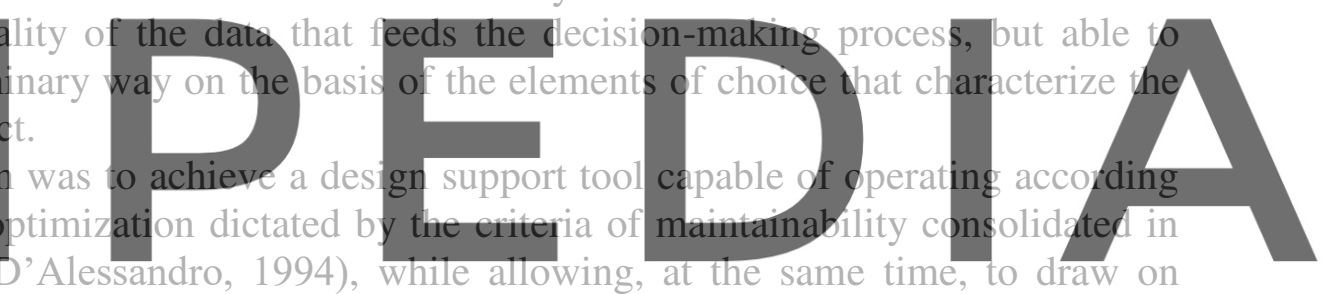

databases, existing or gradually implementable, relating to: incompatibility of materials,

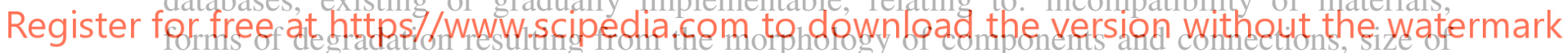

maintenance equipment, ergonomic schemes related to maintenance operations, etc.

In order to be easy to use, the SSD was based on the evaluation and expression of maintenance quality through a rating index. From the methodological point of view, the work has been developed in the direction of the construction of a system capable of managing the large number of variables that distinguish maintenance-oriented architectural design from the initial phase.

The current reality of projects is in fact extremely complex, especially when analyzing problems related to maintainability, where the multitude of variables at stake and uncertainty factors characterize the entire life cycle of the project (Talamo, 2019). It is therefore necessary to be able to use a "interrogable" modelling tool capable of providing support for the automation of evaluation processes formalized on the basis of maintenance criteria that can be applied to the entire architectural organism or to a part of it, such as a façade system. A quantitative approach to integrate uncertainty and the multitude of variables in an objective and rigorous way into reasoning can be implemented through the use of the so-called Bayesian Networks.

The conceptual model for the decision support system has in fact been developed through 
the construction and implementation of a Bayesian Network, a representation of a probabilistic inference model capable of describing the set of stochastic variables and their conditional dependencies through the use of a Direct Acyclic Graph (DAG) (Novi, 2018).

Following a "data driven" strategy, a space-time analysis tool has been developed to support decisions in order to reach a maintainability index. First of all, the knowledge network was built (directly through the Hugin Expert software) by defining a conceptual model consisting of nodes (variables) that represent the "maintainability criteria" defined, then linked together according to criteria of causal dependence (cause - effect).

In order to simplify the operational phase of compiling the values of the variables of the individual nodes - variables, a simple tabular interface has been created with the help of a spreadsheet that makes the input of data by the designer clear and simple and automates the numerical output with a compilation and update link directly to the nodes of the BN created in the Hugin Expert software.

Once the available values are propagated, the network allows to obtain through the expression of a synthetic index, a rating about the maintenance quality of the described project and to perform in real time multi-scenario simulations, diagnostic evaluations, sensitivity analysis, simply by directly modifying the values in the network or in the tabular interface. The tool thus obtained, even in the presence of partial or inhomogeneous data, allows to perform a multitude of simulations and obtain in real time functional results at the level of knowledge of the problem, guiding the designer in a conscious way in the evaluation and the necessary choices throughout the lifecycle desig

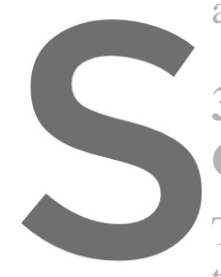
S 3 Implementation of the DSS on a Case Study: 'Th
Groupe
The method described has been tested through the applicat technologies and construction methods with the intent to test the flexibility and compliance of Register for project support tool developed to operate in Gases of uncertainty and complexity, the watermark Here is described the analysis. carried out on a well-known existing building: the headquarters of ING Groupe in Amsterdam (Fig.1), built by Meyer and Van Schooten characterized by a high design attention to the maintenance theme, in order to carry out the evaluation by simulating different possible scenarios related to different technical solutions as may happen in a possible design process. In the experimentation, the maintainability of the building has been tested in three areas: vertical closure - plant engineering system - upper closure but is exemplified here for exposure limits for the evaluations related to the facade.

ING Group is an example of sustainable, innovative and energy-efficient architecture.

The glass and aluminum structure of the building is raised on metal pillars whose height varies between 9 and $12 \mathrm{~m}$. The façade consists of two layers of glass spaced apart which contain a passage to allow both access for maintenance and natural ventilation.

The external glass is hooked through 6 fixing points that guarantee the support of the façade by means of glass elements (thickness $20 \mathrm{~mm}$ ), bound to the floor by stainless steel components. The sheets of $1800 \times 3300 \mathrm{~mm}$ size, are made of tempered or tempered laminated glass 12 or $19 \mathrm{~mm}$ thick. 


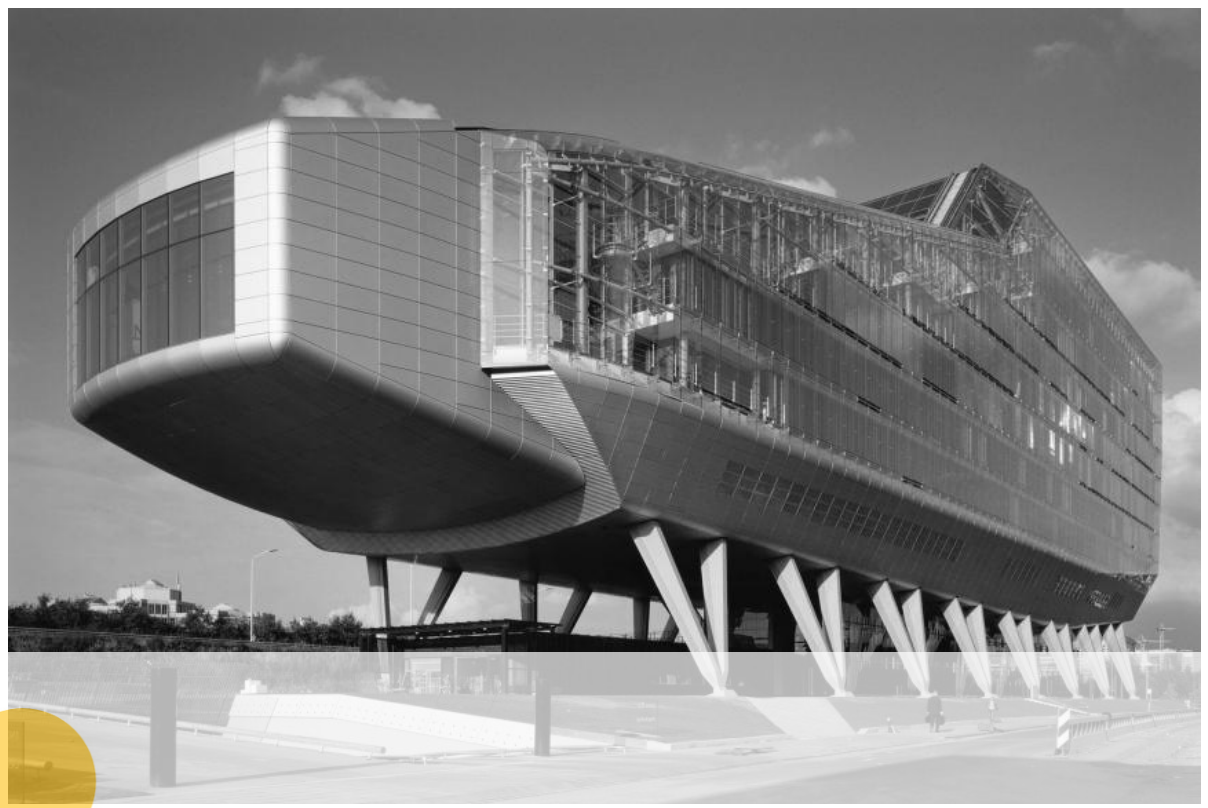

Figure 1. Headquarters ING Group, Amsterdam. MVSA Architects.

The sheets are separated vertically by silicon joints of $12 \mathrm{~mm}$; horizontally by a glass opening element with a height of $410 \mathrm{~mm}$ for the ventilation of the facade. The internal facade is made up of inward opening wooden frames $950 \mathrm{~mm}$ wide and $2900 \mathrm{~mm}$ high. The
cleanability and periodic control of the facade cladding is also guaranteed thanks to the
accessible roof on the tenth floor. A system of tracks that runs through the entire building is
designed to ensure a high level of inspection and maintenance: thanks to the installation of
mobile trolleys on the roof, the easy cleaning of all the windows has been ensured.

In the experimentation of the decision support system, the building was analyzed

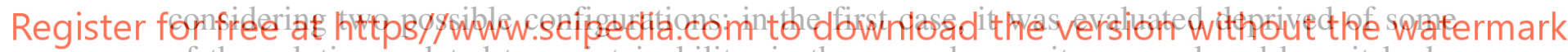
of the solutions related to maintainability; in the second case, it was analyzed how it looks today, with the current technical characteristics related to maintainability

The flexibility of the method, allows to perform multi-scenario analysis by intervening on probability distributions, i.e. to verify how simulations in real time, allow to take strategic decisions. Therefore, each case has been subjected to a double analysis: in the first one the method is tested as a tool for evaluating the building, while in the second one its potential as a support system for decision making has been verified.

The maintainability index of the building is obtained from the analysis of three guiding criteria:

- MATERIALS for assessing the durability not only of the intrinsic properties (quality of the component), but also in relation to the design and execution techniques and the environmental context to which it belongs (design quality - execution quality - environmental quality);

- the MORPHOLOGY OF SYSTEMS for the determination of the morphological quality through the analysis of the sub-requisites of maintainability such as: durability - not dirtiness self-cleaning capacity - cleanability, in order to make more appropriate choices during the design phase to avoid possible pathologies; 
- EASE OF INTERVENTION for the determination of accessibility and spatial ergonomics through the evaluation of the sub-requirements of maintainability such as: maintainability by the user - serviceability - serviceability - reparability - disassembly replaceability.

\subsection{Analysis of Cases and Evaluation of Alternatives}

CASE 01 assumes the construction of the curtain wall using insulating glass on a single layer and without the equipment and access routes to the envelope.

Analysis 01: the first step consists in the realization of three tables on a spreadsheet (information database) in which the parameters of the sub-requirements related to the maintainability of the façade are analyzed, in order to determine the three criteria of the system (durability of the materials - morphology of the systems - ease of intervention) in relation to the materials and components used for the realization of the proposed case study.

The second step provides, in order to obtain the evaluation of the maintainability index, the use of Bayesian Networks. This consists in the realization of a second grid (Fig. 2) that allows to translate the parameters on Hugin (software for the use of Bayesian Networks).

In the third step, the saving of each parameter under examination and the subsequent export to Hugin, implements an automation process at the end of which you get a node for each parameter exported.
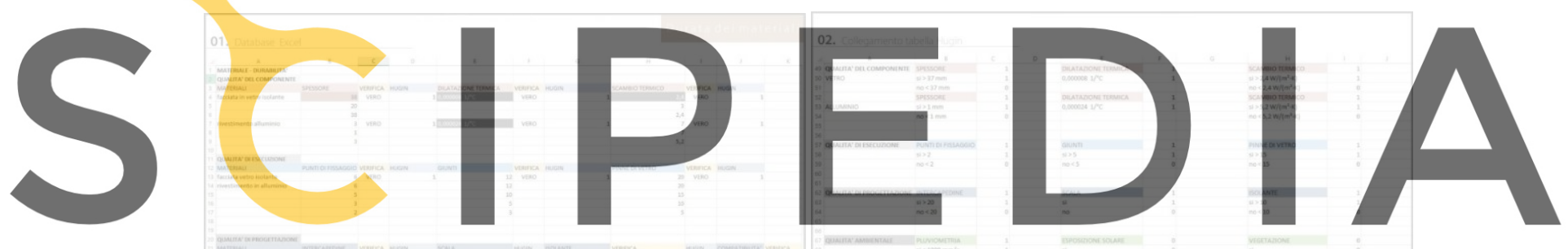

Register for free at https//www.scipedia.com to download the version without the watermark

Figure 2. Database construction and data link.

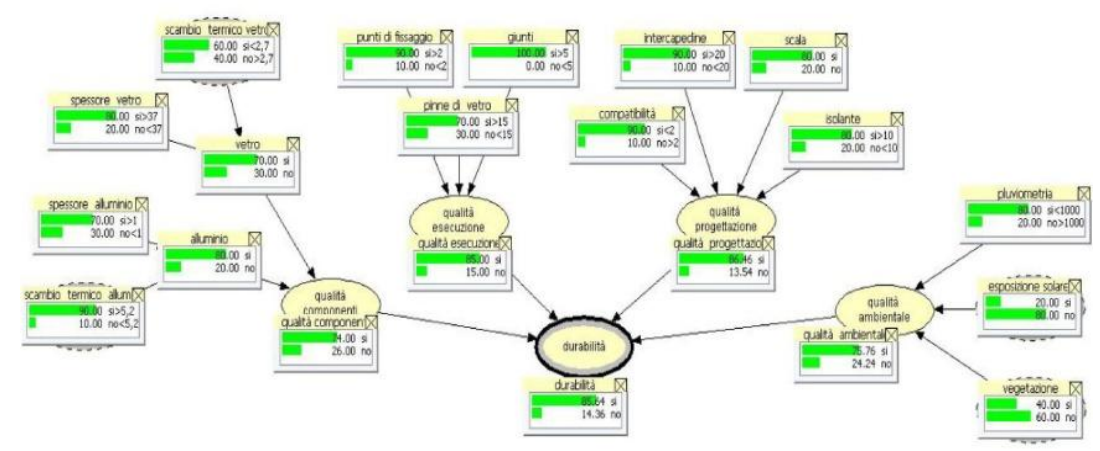

Figure 3. Each node-parameter is linked to a reference table with Boolean values. 
The result is a relationship network (Fig.3) in which each node-parameter is linked to a reference table with values 1 or 0 , indicating whether or not the requirement previously verified on the spreadsheet has been met. From the connection between the nodes it is possible to determine the maintainability of the facade under examination, which in this case expressed an index for maintainability of $48 \%$.

The results obtained show that the main cause is to be attributed to the ease of intervention.

The system of vertical closures and the components integrated in it must be structured in such a way as to make their inspection easy. During the design, especially in relation to the surface conformation of the materials, dust and atmospheric pollution, it is necessary to provide easy access structures to facilitate cleaning operations.

In the Analysis 02, the implementation of a series of operations allows to verify the compliance of the instrument as a support to decision-making choices.

In intervention $\mathrm{A}$ the insertion of paths to overcome the difficulties connected to the periodic cleaning of the curtain wall was foreseen. The integration of this aspect has led to a $50 \%$ increase in inspectability, with an improvement also in terms of the cleanability of the facade.

Intervention B, consisted in the insertion of equipment (ladders - trolleys), particularly recommended for buildings with curtain walls, as they facilitate periodic cleaning and maintenance operations, avoiding the use of particularly expensive external means with consequent time savings. The integration of this aspect has led to reparability from 40 to $80 \%$.

Finally, intervention. C considered all ergonomic aspects with the aim of improving user satisfaction and the overall performance of the system. The intervention concerned the
relationship between the dimensions and the geometry of the operating space, in order to take
into account the body and limb positions of the operators and their movement in relation to
the specific maintenance actions and the use of the required tools and utensils. The ergoromic
aspect, therefore, allows to perform those periodic cleaning and maintenance operations
without which the use of external means would be required.

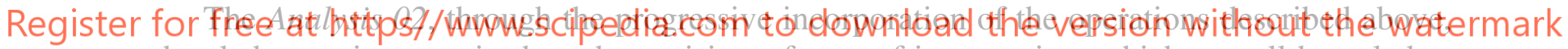
has led to an increase in the sub-requisites of ease of intervention, which overall have led to an increase in terms of maintainability of $15 \%$ (from 48 to $63 \%$ ).

In CASE 02, the insulating glass facade consisting of a transparent double skin was analyzed, with the presence of the inspectionable cavity, the accessible staircase and the tracks that allow the passage of the trolleys throughout the casing.

At the end of the process already described for the previous case here, the Evaluation 01 provided a rating index for maintainability of $71 \%$. Therefore, action was taken where the system had the lowest rating value, which in this case, among the three criteria examined, was the morphology of the systems.

During the design phase it is necessary to pay particular attention to the internal and external factors of the system in order to minimize the inconveniences due to the degradation caused by atmospheric agents on the walls. It was therefore considered, in this case, to provide a geometric configuration of the façade and a morphology of the components such as to allow a homogeneous washout of the surfaces while avoiding stagnation, accumulation, water absorption and the fixing of dust. Therefore, the 02 analysis simulated different conditions on some aspects related to the morphology of the system. 


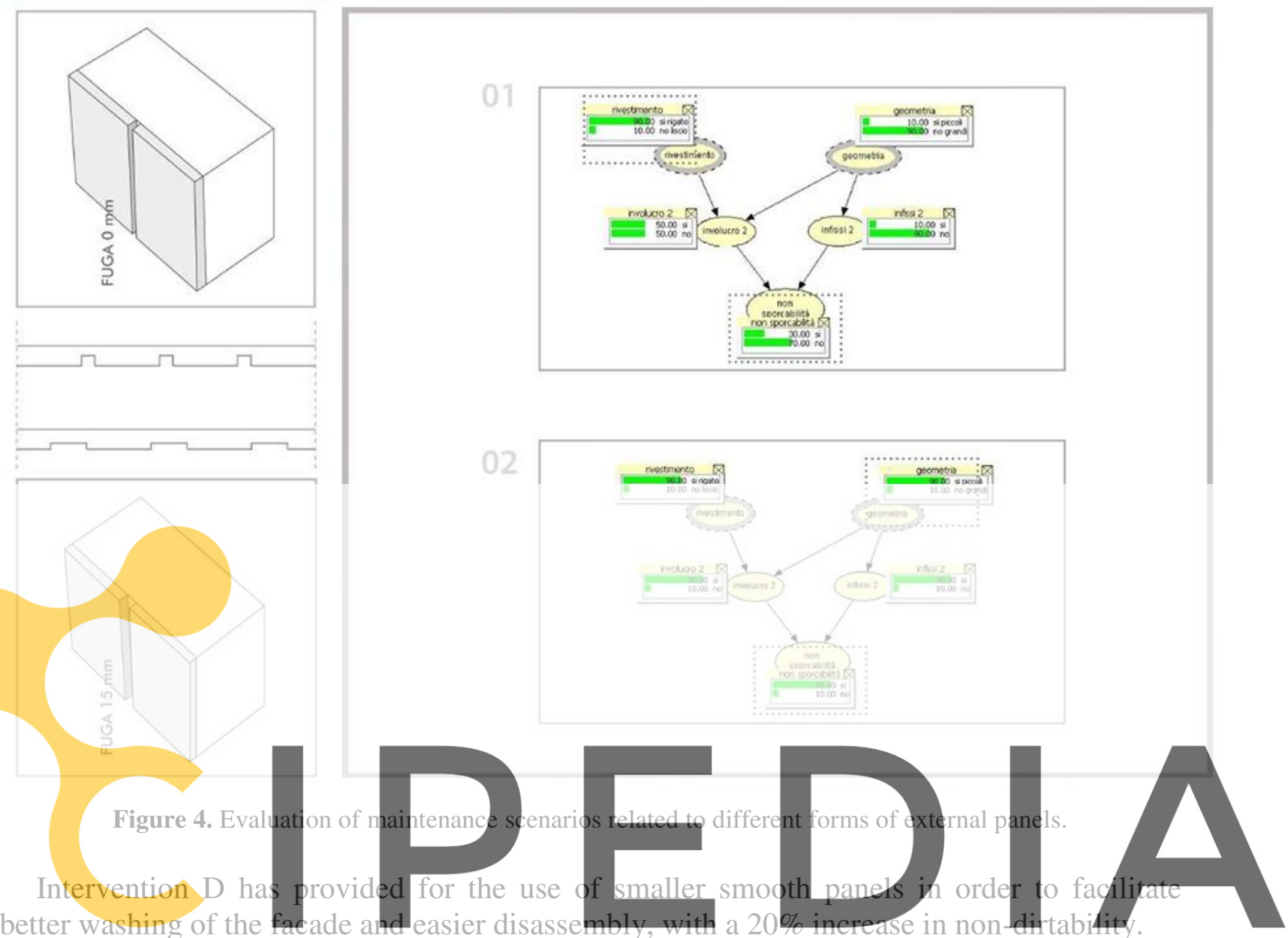

Intervention E assumed the use of striped panels, with sufficiently wide and shallow joints

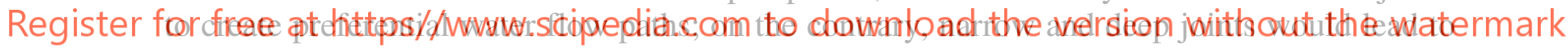
the deposit of debris and dirt with the need for specialized personnel for periodic cleaning operations. This intervention has led to 30 to $90 \%$ non-soiling.

The example described denotes how the Bayesian Networks offer the possibility to verify in real time how much each single intervention can affect, in percentage - probabilistic terms, the degree of maintainability of the project.

In the second analysis of Case 02 of ING, the progressive incorporation of the components led to an increase in the sub-requirements of the morphology of the systems, which on the whole led to an improvement in the maintainability of the system with an increase of $10 \%$ more (from 71 to $81 \%$ ).

The results obtained from the proposed analyses show that certain choices, made during the design phase, can have a high impact on maintainability. Although it is a simple qualitative evaluation, the analysis highlights not only an objective comparison between the two scenarios, but also clarifies and makes explicit all the variables that significantly affect the results. 


\section{Conclusions}

The use of Bayesian Networks, in the light of the analysis carried out on an experimental basis, for the ability to control a multitude of factors related to the durability of materials, the morphology of the systems and the ease of intervention, seems capable of generating a valuable tool not only useful as a support in decision-making ex-ante in the project phase of new construction but also ex-post for the identification of rehabilitation interventions in existing buildings. The system developed allows to determine the impact that determined variables, chosen from the point of view of maintainability, bring to the whole project allowing to establish what attention should be paid to the estimates of probability to obtain certain services of interest or at the same time what corrective actions are essential to be taken.

Future developments in the research of the SSD model are oriented in the direction of the integration of cost parameters for a more complete and effective evaluation and comparison of alternative intervention scenarios and the identification of implementable protocols for an objective comparison between contractors during the awarding of a contract.

\section{ORCID}

Michele Di Sivo: http://orcid.org/0000-0002-2140-9513

Daniela Ladiana: http: // orcid.org/0000-0001-6008-1365
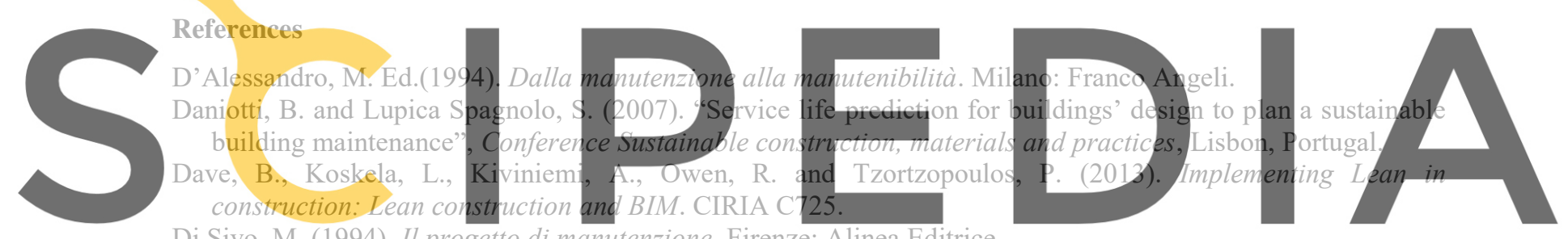

Di Sivo, M. (1994). Il progetto di manutenzione, Firenze: Alinea Editrice.

Di Sivo, M. and Ladiana, D. (2010). "Building maintenance: a technology for resource conservation", WIT

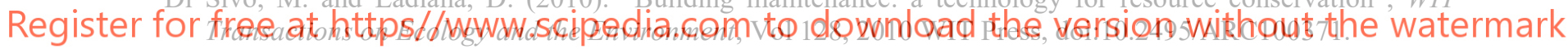

Ladiana, D. (2009). "Durata e sostenibilità del ciclo di vita degli edifici. L'importanza dell'approccio maintenance-oriented." Manutenzione Tecnica e Mangement, anno XVI, n.3, (Marzo)

Latouche, S. (2015). Usa e getta. Le follie dell'obsolescenza programmata [Use ant throw. The Madness of Planned Obsolescence], Torino: Bollati Boringhieri

Novi, F. (2018). "Bayesian networks as a resilience tool for decision - making processes in uncertainty conditions", TECHNE - Journal of Technology for Architecture and Environment, Vol. 15, p 341-347.

Talamo, C., et al. (2019). "Incertezza epistemica, gestione dei rischi e informazione: il ruolo del progetto esecutivo". TECHNE - Journal of Technology for Architecture \& Environment. Vol. 18, p164-173. 